\title{
Challenges and controversies in COVID-19 respiratory failure
}

\author{
Simran Kaur Matta MD
}

\begin{abstract}
COVID-19 pneumonia presents distinctive questions and challenges traditional conventions of management of respiratory failure. The trajectory of recommendations on customary intubation practices has undertaken significant paradigm shifts. This review will discuss the role of high flow nasal cannula oxygenation in mitigating respiratory distress in SARS-COV2 pneumonia and will explore the indices that can aid in the timely recognition of failure of noninvasive respiratory support modalities and escalation to mechanical ventilation. The work of breathing is a valuable yardstick for understanding increasing lung elastance. Quantifying work of breathing, though, has its own unique challenges. This article also discusses the emerging controversial proposals of employing high tidal volumes and low PEEP in mechanical ventilation of COVID-19 pneumonia and will review the key concepts of lung stress and strain and the implications of "static" versus "dynamic" strain in ventilator induced lung injury. It considers the established facts of inducing lung strain with larger dynamic deformations caused by high tidal volumes and the benefit of high PEEP in homogenizing the strain distribution. The review suggests that the isolated ground glass opacities could pose as "stress raisers." The effects of these regional lung homogeneities in amplifying local and global lung stress are also discussed as well as the benefits of PEEP beyond its effect as a pressure barrier against alveolar filling and its utility in lungs with near normal compliance. A physiologic approach is presented to counter the non-uniform and heterogeneous presentations of this unique disease rather than conforming to rigid protocols. One size probably does not fit all.
\end{abstract}

Keywords: Covid-19, early intubation, patient self-induced lung injury (P-SILI), pendelluft, lung stress/strain, $\mathrm{H}$ and $\mathrm{L}$ phenotypes, high tidal volume, low PEEP

\section{Discussion}

Significant institutional planning and several logistical challenges, e.g., transport to a negative pressure room and vigilant infection control, have led to support of early, non-emergent intubation. An inability to adequately pre-oxygenate due to curtailment of bagging and the use of non-invasive positive pressure ventilation (NIPPV) have also resulted in early intubation. However, such practice patterns may not be sustainable

Corresponding author: Simran Kaur Matta Contact Information: Simran_Kaur_86@hotmail.com DOI: $10.12746 /$ swrcc. v8i35.743 given the limited resources nor are they necessarily best practices. While establishing safer and more reliable guidelines to circumvent emergency intubation is necessary, how does one decide on the correct timing while avoiding intubations that are unwarranted?

COVID-19 has reminded healthcare workers to reconsider the basics of critical care medicine-when to intubate?

COVID-19 pneumonia is anything but uniform. "Happy" hypoxemia (significant hypoxemia without dyspnea), while not a new phenomenon, is being encountered more in COVID-19 pneumonia, and there has been clinical debate on early versus late intubation. "Happy hypoxics" can stay on high oxygen levels 
using high flow nasal cannulas (HFNC) for days before they begin improving. The therapeutic challenge is whether or not the respiratory failure can be managed without recourse to an endotracheal tube.

Two distinct phenotypes and pathophysiologic entities have been suggested. Gattinoni et al. have referred to them as " $\mathrm{H}$ " and " $\mathrm{L}$ " phenotypes. ${ }^{1}$ Some authors prefer "compliant" and "stiff" lungs. These presentations are by no means mutually exclusive and often present as a continuum in the spectrum of SARS-CoV-2 respiratory failure. With relatively preserved lung compliance, impaired regional vasoplegia and loss of compensatory vasoconstrictive mechanisms contribute to low V/Q ratios, the underlying mechanism postulated to explain the initial hypoxemia in the L phenotype. Conventional oxygen therapy and HFNC are typically the next steps in the escalation of respiratory care in these patients. Without significant lung volume available for recruitment using PEEP, the benefits of the application of PEEP with the use of non-invasive positive pressure ventilation (NIPPV, e.g., CPAP or BiPAP) balanced against the risks of aerosolization using these modalities have been questioned. While some may have an indolent course, some patients can progress to a typical ARDS lung, an "H" phenotype. Patients with prolonged symptoms may present to the acute care setting with an $\mathrm{H}$ type. With transition to the $\mathrm{H}$ phenotype, multiple lung units begin to collapse and remain unavailable for gas exchange. Dead space leads to inefficient $\mathrm{CO}_{2}$ clearance, so larger tidal volumes are necessary to maintain a physiologic $\mathrm{CO}_{2}$ level. Higher values of $\mathrm{pCO}_{2}$ increase the respiratory drive to achieve the necessary increases in minute ventilation. The greater the dead space, the higher the necessary minute ventilation. An increase in minute ventilation requires an increase in the work of breathing. The work of breathing during the inspiratory limb is further increased due to the increase in lung elastance. This combination of ineffective $\mathrm{CO}_{2}$ clearance, impaired gas exchange, and stiff lungs leads to dyspnea and increased work of breathing. ${ }^{2}$

High oxygen requirements alone should not be the deciding factor for intubation. Several recent patients required $\mathrm{FiO}_{2}$ upward of $0.8-1.0$, but at some point, the disease seemed to "turn off," and the patients began recovering with successful weaning of oxygen requirements. This was also noted in a recent paper by Fernandez et al. ${ }^{3}$

It is crucial to prevent the progression to an $\mathrm{H}$ phenotype, i.e., the typical ARDS lung or "stiff" lungs. In non-intubated patients, it can be difficult to assess for recruitability and lung elastance. Computed tomography (CT) scans can give insight into identification of these two phenotypes and an estimate of recruitable lung volume. However, one needs to be careful inferring recruitability from a single, static CT scan. Establishing biomarkers for recognizing the necessity for intubation as well as for early detection of transi-tion from "compliant" to "stiff" lungs is important.

Work of breathing (WOB) can be a surrogate marker of compliance of the lungs. Let us consider the physiology and first define the work of breathing. Work is force applied over a distance. The work of breathing is the volume integral of pressure over an inspiratory cycle $(\mathrm{dW}=\mathrm{PdV})$. The total work for a single breath across the lung can be subdivided into two compo-nents. The first component is the pressure required to overcome the elastance of the lung and chest wall $(\mathrm{dP}=$ $\mathrm{EdV}$ ). As this is the pressure dedicated to increase the volume of an elastic structure, it is called "elastic" work. This can be further subdivided into PEEPrelated elastic work and tidal volume-related elastic work. The second component is the pressure required to overcome the resistance of the airways to allow airflow. This is also called "resistive work" ( $d P=R d V / d t)$. Resistance is the derivative of pressure with respect to flow, and flow is the time derivative of vol-ume. In clinical literature, the elastic and resistive work are sometimes referred to as "static" and "dynamic" work, respectively. Elastance is the derivative of pres-sure with respect to volume, and volume is not static during the delivery of a breath. Work, by definition, is not static and requires displacement. At the end of inspiration, the flow is zero, and all the pressure is due to elastance, but that doesn't make it static, because during the inspiration, some of $\mathrm{dP}$ is employed towards $\mathrm{dV}$ and some is applied to $\mathrm{dV} / \mathrm{dt}$ which is necessary for $\mathrm{dV}$ $>0$. Thus, we will avoid the use of "static" and "dynamic" work nomenclature in further discussions.

Work of breathing should serve as a useful clin-ical tool to assess failure of non-invasive respiratory 
support and progression to a typical ARDS lung. Can its evaluation be standardized? Tobin has pointed out some useful physical signs: palpation of the sternomastoid contraction, tracheal tug, inspection of the suprasternal fossa and intercostal spaces for recession, and the presence of diaphoresis. ${ }^{4}$ It is necessary to quantify these metrics, grade the qualitative data, and account for inter-observer variability. Frequent and close contact physical examinations are being curtailed during this pandemic to minimize contact and to ration the use of PPEs. Clinicians sometimes rely on respiratory rate as a gauge for respiratory distress, but there is not a threshold breakpoint that triggers intubation. It is incorrect to solely regard tachypnea as a sign of increased work of breathing; instead, WOB is determined by the magnitude of pleural pressure swings and tidal volume. And more important, all of the above need to be interpreted in the clinical and physiological context. If the patient has respiratory acidosis but no significant distress, it still suggests failure of respiratory function.

While assessment of WOB is an important sign of respiratory depression and is a key indicator for intubation, it is necessary to rely on the physician's clinical acumen, experience, and wisdom. Gattinoni et al. suggested the use of esophageal manometry for the detection of excessive inspiratory effort. Interpretation of esophageal pressure tracings, though, is not a common skill and can be daunting for many physicians. Also, it is unknown how to interpret these numbers in a prone patient.

The use of high flow nasal cannulas is significant for their role in the mitigation of mechanical power to the lung, i.e., work of breathing over time. The WOB is a combination of resistive work and elastic work. A high flow nasal cannula, set at $60 \mathrm{~L} / \mathrm{min}$, significantly reduced the measured indexes of respiratory effort, such as esophageal pressure variation, esophageal pressure-time product/minute, and work of breathing/ minute. These results reached clinical and statistical significance. The WOB was noted to fall by $50 \%$ along with an increase in dynamic lung compliance and a decrease in inspiratory resistance. This was a small study with 12 patients in moderate distress who were recovering from an acute episode ${ }^{5}$ while these findings should not be directly applied to all patients with an acute episode, there may be a role for HFNC in mitigating mechanical power and patient self-inflicted lung injury (P-SILI) at the earliest signs. During the early phase of transition to the $\mathrm{H}$ phenotype, before significant respiratory distress sets in, HFNC may have a role in reducing the amount of physiological and anatomical dead space, thereby reducing the respiratory drive and minimizing progression to P-SILI.

Although P-SILI is a controversial topic, it bears on the transition to a typical ARDS lung. Excessive negative intrathoracic pressures from vigorous breathing efforts can generate potentially injurious transpulmonary pressure swings. In normal lungs, local changes in pleural pressure are generalized over the entire pleural surface. In patients with existing lung injury, negative forces generated by the respiratory muscles may lead to injurious regional effects. In addition, the increase in transmural pulmonary vascular pressure swings caused by inspiratory effort may increase vascular leakage. ${ }^{6,7}$ Furthermore, strong spontaneous respiratory efforts in an already injured lung can cause pendelluft (an intra-tidal shift of gas between different lung zones) due to more localized changes in pleural pressures generated by different transmission of muscular forces that are not distributed uniformly. ${ }^{8}$ This concept parallels ventilator-induced lung injury. Could VILI also translate to ventilation-induced lung injury?

Some clinicians have considered P-SILI to be a fragile concept given the lack of methods to measure and demonstrate it, but one could argue it has a strong physiological basis. Clinicians managing non-intubated COVID-19 patients who are progressing to ARDS should be aware of the risk of P-SILI and monitor closely for escalation of respiratory support. Self-induced lung injury is especially relevant in the current pandemic situation, which is resource constrained. If intubated after significant worsening has occurred, the patient may be subject to a rather prolonged period of mechanical ventilation.

It is not practical to obtain frequent CT scans or chest $\mathrm{x}$-rays to follow the progression of disease. It is worthwhile reviewing the utility of lung ultrasound as an effective means to evaluate the extent of the disease and to recognize the evolution to an ARDS 
pattern. Involvement of multiple lung quadrants, an increasing number of B lines, coalescence of B lines, white lung, worsening pleural irregularities and interruptions, and an alveolar consolidation pattern are key signs to disease progression and diffuse involvement of pneumonia. ${ }^{9}$

Rapid shallow breathing in itself can decrease alveolar ventilation by increasing the physiologic dead space and ultimate fatigue of the respiratory pump. The benefit of HFNC in counteracting a progressively enlarging oxygen debt and averting intubation should be applied on a case-by-case basis. The lack of timely interventions in patients with respiratory distress and delays in intubation can escalate into worse outcomes and prolonged intubation periods. Rather than utilizing a certain oxygen requirement as a threshold, the decision to intubate must be tailored to the individual's pathophysiology. Hypoxia alone, without accompanying respiratory distress or severely abnormal gas exchange, does not require escalation to mechanical ventilation. In fact, $\mathrm{PaCO}_{2}$ levels provide a greater insight into respiratory impairment and changes in minute ventilation. Similarly, the sole presence of extensive infiltrates that are being managed with supplemental oxygen does not constitute a reason for intubation. Patterns determined by physiologic parameters, sound clinical assessment of work of breathing, utilization of lung ultrasound, identification of and timely intubation of patients who are at risk of progression to ARDS are likely to yield superior outcomes.

Another paradigm shift in the management of SARS-CoV-2 respiratory failure is the resurgence of high tidal volume and low PEEP ventilator settings, first proposed by Gattinoni and colleagues in the $L$ phenotype subset. However, their current proposal refutes their own studies and is at odds with years of research in protective ventilation strategies. Gattinoni et al. had noted in their earlier studies that incremental strain due to high tidal volumes is more injurious than continuous strain from high PEEP. ${ }^{10}$

Again, let us first define some key concepts and terminology. Stress is a spatial derivative of force over an object having dimensions rather than a point. Stress leads to deformation or displacement of the object. Strain is the spatial derivative of the deformation. In simpler words, stress is force per unit area, and strain is the deformation of the material as a result of stress. In a linearly elastic body, the stress and strain have a linear relationship. In a viscous substance, there is additional stress caused by the spatial gradient of the velocity of flow. Thus for a viscous material, strain is not only a function of stress, but is also a function of the spatial distribution of stress as well and fluctuations of stress over time. From a biomechanical viewpoint, lungs behave as viscoelastic substances, combining the properties of both of viscous and elastic bodies.

In clinical literature, often times, stress/strain as a result of applied tidal volume is referred to as "dynamic" and stress/strain from applied PEEP is referred to as "static." However, one would argue that this nomenclature is not entirely correct. During the process of deforming, the motion of the object is not uniform due to viscosity. Some of the stress generates motion and viscous drag, while some of the stress is necessary to maintain the deformation. This is analogous to the elastic component of pressure and the resistive component of pressure, but it is not the entire picture due to nonhomogeneity. Stress is the spatial derivative of force. So, a non-homogeneous lung has alveoli with different wall tensions even when the pressure is uniformly distributed. More compliant alveoli have larger stresses than stiffer alveoli at each lung volume. Furthermore, the more compliant alveoli have larger $\mathrm{dV}$ for each $\mathrm{dP}$, so there is greater stress necessary to overcome viscous drag during inflation. The stress between interdependent alveoli has a component due to elastic force and component due to viscous drag. The elastic force is not truly static as it increases with lung volume during inspiration. However, since "dynamic" and "static" terms are so often in clinical research, we could work a way of defining and utilizing them for the purpose of the current discussion. Static stress would have to be defined as continuous stress applied over the entire respiratory cycle by PEEP. Static strain would have to be defined as the deformation resulting from static stress. Dynamic stress would be the fluctuating stress due to tidal volume repeating with each respiratory cycle. Dynamic strain would be the deformation resulting from dynamic stress. The dynamic portion of the signal is the fluctuation about the average value. The static portion of the signal is the average value. 
Lung deformation from tidal ventilation yields dynamic or incremental strain. The application of PEEP to a non-homogeneous distribution of elastance leads to a deformation or strain that is continuous over the respiratory cycle. Total strain is the sum of the strain continuous over the respiratory cycle and the incremental strain that fluctuates during the respiratory cycle. The current clinical surrogates of lung stress and strain are plateau pressure and tidal volume as standardized on ideal body weight. In investigating the effects of dynamic and static deformation by ventilating with varying combinations of tidal volume and PEEP, Protti et al. found that dynamic strain above $>2.0$ with resultant stress $>15 \mathrm{cmH}_{2} \mathrm{O}$ invariably caused fatal pulmonary edema. By contrast, the same level of total strain induced mainly by PEEP ended the experiment with normal lungs. This answers a crucial question regarding the nature of the stress applied. The threshold of total strain and overall inflation of the lungs are not the sole determining factors of lung injury; the manner in which it was achieved also holds significance. ${ }^{10,11}$ Due to the tissue viscoelasticity, large static strain is better tolerated than equivalent dynamic inflation.

Would the time-tested principles of limiting large deformations by restricting tidal volumes and diminishing lung inhomogeneities by static inflation with PEEP fly in the face of L-type ARDS? COVID-19 respiratory failure is rather the rule, not the exception.

Again, remember that the lung is also viscous. Thus, a component of lung strain is proportional to the velocity of deformation and is higher during air flow. ${ }^{12,13}$ A greater volume change per unit time, as with higher tidal volume, can augment the stress and hence the risk of rupture across the viscous lung. While the mechanisms of transformation of nonphysiological mechanical force by means of large stress and strain into a biological reaction remain ill defined, evidence shows that these forces invoke inflammatory pathways and lung injury. ${ }^{14}$ Tidal inflation patterns that employ small and slow cyclic strain rather than large and rapid deformations are crucial to maintaining the integrity of the pulmonary micro-architecture. Application of PEEP is currently the primary strategy by which to minimize dynamic strain for established ARDS.
One argument posits that since there isn't much lung to recruit, there might be no additional benefit to using high PEEP and that high PEEP only begets worsening hemodynamics status. It is imperative to remember that PEEP is not merely a hydrostatic barrier against edema formation. There are other mutually non-exclusive explanations why PEEP is useful in lung protective strategies. Factors other than total endinspiratory stress and strain need to be considered when implying the advantages of PEEP in a lung with relatively normal compliance, such as possible presence of inhomogeneous areas and "stress raisers."

While there may be larger proportions of disease-spared normal lung tissue, wouldn't the isolated areas of ground glass opacities act as "stress raisers" or "pressure multipliers?" Wouldn't the focal inhomogeneities outset the VILI vortex?

The mechanical interdependence of the air spaces in a viscoelastic substance causes amplification of stress in a non-homogeneous lung, with significantly higher stress borne at the interface of closed and open lung units. ${ }^{15}$ Even though the focal ground glass opacities may involve relatively small lung regions, they can act as "stress raisers" or "pressure multipliers" and initiate the process of VILI. This effect is further amplified at the interface of the injured and normal lung regions. Rausch et al. studied this phenomenon extensively and concluded that stress raisers can amplify the local stress up to four times. ${ }^{16}$ PEEP also appears to reduce inflammation, and, in fact, ZEEP (zero end-expiratory pressure) has been shown to cause a greater increase in inflammatory mediators than PEEP. ${ }^{17}$

In patients who have not progressed to ARDS and have greater functioning lung volumes, use of higher tidal volumes has provoked discussions. In a tidally recruited lung, there are proportionate increases in the volumetric strain incurred at higher tidal volumes applied. Paula et al. analyzed this effect in computing the effects of different levels of tidal volumes and PEEP on lung strain and risk of VILI. They noted that increases in tidal volume resulted in spatially heterogeneous increases in lung strain, with maximum volumetric strain occurring at mid and dependent lung regions, implying topographic variation in risks of strain 
even within a normal lung parenchyma. PEEP, on the other hand, was beneficial in mitigating the strain from tidal recruitment, homogenizing the spatial distribution of regional strain and reducing inflammation. ${ }^{18}$ This is also important when estimating the volume of a deformed lung, i.e., de-recruited lung tissue and the area at risk of VILI. It also suggests how to define recruitability. Is it simply the size of lung tissue that has been deformed from its normal anatomy? The extent of functionality of these deformed areas as right-toleft shunts, their topographical distribution, and risk of lung strain and injury could have plausible effects.

While ventilating with volumes $9 \mathrm{ml} / \mathrm{kg}$ ideal body weight (IBW) may not be appropriate, applying 4-5 ml/ $\mathrm{kg}$ IBW tidal volume may increase dead space ventilation since it is not a truly "baby" sized lung volume. Tidal volume titration based on markers reflective of the severity of lung injury, including driving pressures and compliance, is probably the most effective means of ventilating all phenotypes of COVID-19 pneumonia. Ideally, developing systems of measurement of available "baby lung volume" and strain metrics would provide an improved physiologic approach to ventilation.

In severely hypoxemic patients requiring $\mathrm{FiO}_{2}$ 0.9-1.0, should PEEP upward to $15-20 \mathrm{~cm} \mathrm{H}_{2} \mathrm{O}$ as per the ARSDnet protocol be applied in cases with rather compliant lungs? High PEEP is paramount, but how high is the appropriate and tolerable PEEP in the L-type so as to counterpoise the benefits of alveolar stability and strain homogenization with the harms of overinflation of the larger available healthy lung units? Multiple methods of personalizing PEEP have been tested, such as dead space, lung compliance, lung stress and strain, ventilation patterns using computed tomography (CT) or electrical impedance tomography (EIT), and inflection points on the pressure/volume curve $(P / V)$; however, there remains no consensus as to the optimum approach. ${ }^{19}$ This novel respiratory disease does challenge the traditional protocols and poses more questions than answers. Repudiating old doctrines versus adhering to all-encompassing time-tested protocols? While radical changes to existing practices can be controversial, an individualized mechanical ventilation strategy directed by physiological parameters could improve outcomes.
A physiological mindset may invoke greater appreciation for its heterogeneous presentations than the uninspired drudgery of elegant algorithms.

Article citation: Matta SK. Challenges and controversies in COVID-19 respiratory failure. The Southwest

Respiratory and Critical Care Chronicles 2020;8(35):

29-35

From: Bayhealth Hospital, Milford, Delaware

Submitted: $6 / 22 / 2020$

Accepted: $7 / 18 / 2020$

Reviewer: Gilbert Berdine MD, Victor Test MD

Conflicts of interest: none

This work is licensed under a Creative Commons

Attribution-ShareAlike 4.0 International License.

\section{REFERENCES}

1. Gattinoni L, Chiumello D, Caironi P, et al. COVID-19 pneumonia: different respiratory treatment for different phenotypes? Intensive Care Med 2020; DOI: 10.1007/s00134020-06033-2

2. Coccia CBI, Palkowski GH, Schweitzer B, et al. Dyspnoea: Pathophysiology and a clinical approach. South African Med J 2015;106(S.1.):32-36.

3. Villarreal-Fernandez, E., Patel, R., Golamari, R. et al.A plea for avoiding systematic intubation in severely hypoxemic patients with COVID-19-associated respiratory failure. Crit Care 2020;24:337. https://doi.org/10.1186/s13054-020-03063-6

4. Tobin MJ. Why physiology is critical to the practice of medicine: a 40-year personal perspective. Clinics Chest Med 2019; 40(2):243-257. https://doi.org/10.1016/j.ccm.2019.02.012

5. Delorme M, Bouchard PA, Simon M, et al. Effects of high-flow nasal cannula on the work of breathing in patients recovering fromacuterespiratory failure. CritCareMed2017;45(12):19811988. doi:10.1097/CCM.0000000000002693

6. Grieco DL, Menga LS, Eleuteri D, et al. Patient self-inflicted lung injury: implications for acute hypoxemic respiratory failure and ARDS patients on non-invasive support. Minerva Anestesiol 2019;85(9):1014-1023.

7. Arnal JM, Chatburn R. Paying attention to patient selfinflicted lung injury. Minerva Anestesiol 2019;85(9):940-942.

8. Yoshida T, Roldan R, Beraldo MA, et al. Spontaneous effort during mechanical ventilation: maximal injury with less positive end-expiratory pressure. Crit Care Med 2016;44(8); e678-e688. doi: 10.1097/CCM.0000000000001649. 
9. Volpicelli G, Gargani L. Sonographic signs and patterns of COVID-19 pneumonia. Ultrasound J 2020;12:22. https:// doi.org/10.1186/s13089-020-00171-w

10. Protti A, Votta E, Gattinoni L. Which is the most important strain in the pathogenesis of ventilator-induced lung injury: dynamic or static?, Curr Opin Crit Care 2014;20(1):33-38.

11. Gattinoni L, Carlesso E, Caironi P. Stress and strain within the lung. Curr Opin Crit Care. 2012;18(1):42-47.

12. Bachofen H, Hildebrandt J. Area analysis of pressure-volume hysteresis in mammalian lungs. J Appl Physiol 1971;30: 493-497.

13. Lin YH. Polymer viscoelasticity: basics, molecular theories, experiments, and simulations. 2nd ed.2011; Singapore: World Scientific Publishing, 328-338.

14. Gattinoni L, Protti A, Caironi P, et al. Ventilator-induced lung injury: The anatomical and physiological framework. Crit Care Med 2010;38(10):S539-S548 doi: 10.1097/CCM. 0b013e3181f1fcf7.
15. Mead J,Takishima T, Leith D. Stress distribution in lungs: a model of pulmonary elasticity. J Appl Physiol 1970; 28(5):596-608.

16. Rausch S M K, Haberthür D, Stampanoni M, et al. Local strain distribution in real three-dimensional alveolar geometries. Ann Biomed Eng 2011;39,2835. https://doi.org/ 10.1007/s10439-011-0328-z

17. Chu EK, Whitehead T, Slutsky AS. Effects of cyclic opening and closing at low- and high-volume ventilation on bronchoalveolar lavage cytokines. Crit Care Med 2004;32(1):168174. doi:10.1097/01.CCM.0000104203.20830.AE.

18. Paula LF, Wellman TJ, Winkler $T$, et al. Regional tidal lung strain in mechanically ventilated normal lungs. J Applied Physiol (Bethesda, Md.: 1985) 2016;121(6):1335-1347.

19. Nieman GF, Satalin J, Andrews P, et al. Personalizing mechanical ventilation according to physiologic parameters to stabilize alveoli and minimize ventilator induced lung injury (VILI). Intensive Care Med Exp 2017;5(1):8. Doi: 10.1186/ s40635-017-0121-x 